\title{
Ein Beitrag zur Statistik der Epididymitis gonorrhoica.
}

\author{
Von \\ Dr. Arth. Jordan, \\ ord. Arzt am Mjassnitzki-Hospital zu Moskau.
}

Es gibt zwar eine ganze Reihe statistischer Arbeiten über die gonorrhoische Nebenhodenentzündung, diese häufigste aller T'ripperkomplikationen, aber, so viel ich weib, nur wenige über die Verbältnisse in Rußland. Auberdem hält Fing e $\mathbf{r}^{1}$ ) die Daten über die Häufigkeit der Epididymitis nicht für genau, da die Statistiken dem Hospitalmaterial entnommen sind. Ähnlich äußert sich Schäffer, ${ }^{2}$ ) indem er sagt: „über die Häufigkeit der Erkrankung auf die Gesamtzahl der Gonorrhoen des Mannes berechnet, lassen sich keine genauen Angaben machen, da die zahlreichen diesbezüglichen Statistiken große Differenzen (von weniger als 4 Prozent bis zu fast 40 Prozent) aufweisen". In demselben Sinn spricht sich auch $v . Z$ eis $s l^{3}$ ) aus, da es in seinem Lehrbuch der venerischen Krankneiten heißt: „Die Angabe über die Häufigkeit dieser Komplikation wird von verschiedenen Autoren mit $10-30 \%$ der von ihnen im Krankenhause beobachteten Tripperkranken angegeben. An den Tripperkranken der Privatpraxis, die sich zweckmäßiger verhalten können, tritt die Nebenhodenentzündung relativ seltener auf."

Die Bedenken der Autoren hinsichtlich des Wertes der Krankenhausstatistiken scheinen nur zu berechtigt zu sein, da die Tripperkranken das Hospital meist erst bei schon eingetretenen Komplikationen aufzusuchen pflegen. Aber auch die ambulatorisch behandelten Tripperkranken können nicht ohne weiteres ein richtiges Bild liefern, da es nur einen

1) Finger. Die Blennorhoe der Sexualorgane. Leipzig 1901. p. 293.

2) Lesser. Enzyklopädie der Haut- und Geschlechtskrankheiten. Leipzig 1900. pag. 122.

3) M. v. Zeiss l. Lehrbuch der venerischen Krankheiten. Stuttgart 1902. pag. 107. 
bestimmten, oft verbältnismäßig kurzen Zeitraum ibres Lebens widerspiegelt, denn der Patienten, welche bei venerischen Leiden dauernd sich an einen und denselben Arzt wenden, gibt es nicht viele. Um eine möglichst genaue Vorstellung von der Häufigkeit der Epididymitis zu erhalten, müssen wir viel größere Lebensperioden der Patienten übersehen können, als es bisher bei den Statistiken der Fall war. Diese Lücke auszufüllen, ist nur die Anamnese im stande, welche freilich, insbesondere bei venerischen Kranken recht unzuverlässig angesehen wird. Wenn aber von kompetenter Seite bei viel ernsteren Fragen wie z. B. bei der Tabes auf Grund der Anamnesenerhebung weitgehende Schlüsse gezogen werden, sehe ich nicht ein, warum es nicht für die Epididymitisfrage geschehen kann. Eines Versuches scheint mir jedenfalls die Sache wert, zumal da die akute Nebenhodenentzündung so stürmisch zu verlaufen pflegt, daß sie sich selbst ungebildeten Patienten dauernd dem Gedächtnis einprägt. Andererseits gibt es freilich auch Kranke, welche ihre Leiden gern übertreiben und vorübergehende Hodenschmerzen als Entzündung dieses Organes deuten. Diesen gagenüber bieten die in der Regel nach Epididymitiden nachbleibenden Intiltrate ein sicheres Kriterium. Eine Trennung der Orchitis von der Epididymitis kann selbstredend auf Grund der Anamnese nicht vorgenommen werden, jedoch betreffen die gonorrhoischen Erkrankungen in der Regel zuerst den Nebenhoden und greifen nur gelegentlich auf den Hoden über. Natürlich muß die Möglichkeit zugegeben werden, daß die eine oder andere pseudogonorrhoische Epididymitis bei diesem Modus der Bestimmung mitunteriäuft, auf deren Vorkommen in letzter Zeit mehrfach die Aufmerksamkeit gelenkt worden ist, so durch Boekhart ${ }^{1}$, Elsenber $g_{;}{ }^{2}$ Legrain et Legay, ${ }^{3}$ ) Galewsky, ${ }^{4}$ ) Porges ${ }^{5}$ ) u. a., aber das können nur vereinzelte Fälle sein. Mir ist bloß ein einziger derartiger Fall in der Praxis begegnet. Verwechslungen mit gummöser oder tuberkulöser Hodenerkrankung sind noch weniger wahrscheinlich, weil diese schmerzlos verlaufen und erstere nicht ohne sonstige syphilitische Erscheinungen bleibt, letztere stets zur Operation führt. Eher könnten Verwechslungen vorkommen mit den als Begleiterscheinung infektiöser Krankheiten, wie z. B. Parotitis epidemica oder auch ganz idiopathisch vorkommenden

1) Bockhart. Über die pseudogonorrhoische Entzündung der Harnröhre und des Nebenhodens. Monatshefte für prakt. Dermat. 1886. Nr. 4. Ref. Virchow-Hirsoh 1886. pag. 560.

2) Elsenberg. Gibt es eine idiopathische Nebenhoden- u. Hodenentzündung. Wiener med. Presse 1893. Nr. 31 nnd 32.

$\left.{ }^{3}\right)$ Legrain et Legay. Sur un cas d'uréthritis sans gonococques avec complication d'épididymite. Ann. des mal. genitourinaires 1891. p. 706.

4) Galewsky. Über chr., nicht gonorrhoische Urethritis. Zentralblatt f. die Kr. der Harn- u. Sexualorg. 1903. Bd. XIV. H. 9.

5) F. Porges. Über nicht gonorrhoische, metastasierende Urethritis. Prager med. W. 1903. Nr. 53. 
Hodenschwellungen. Unlängst sah ich einen derartigen Fall, wo bei einem Herrn, im Anschlub an Typhus abdominalis, eine linksseitige Orchitis auftrat, für die eine andere Erklärung fehlte. Das sind aber auch nur Ansnahmeerscheinungen, so daß die anamnestisch erhobene Epididymitis, bei bestehendem oder bei bestandenem und nicht völlig geschwundenem Tripper, mir nicht ohne Bedeutung za sein scheint.

Wenden wir uns nun meinen Beobachtungen zu, so betreffen sie einerseits genau verfolgte Tripperfälle aus meiner Privatpraxis und aus einigen Ambulatorien, wo ich Kranke empfange. Andererseits aber betreffen sie Kranke, welche sich wegen Hautleiden, Syphilis etc. ebendaselbst an mich wandten und nach ihren Aussagen Tripper gehabt hatten. Die von mir in Berücksichtigung gezogenen Ambulatorien gehören an: dem Moskauer Evangelischen Hospital, der Gesellschaft russischer Ärzte auf dem Arbat, der Post und endlich einer kleinen Fabrik. Da ein jedes dieser Ambulatorien vorwiegend von einer bestimmten und von der anderen verschiedenen Gesellschaftsklasse besucht wird, so handelt es sich um ziemlich mannigfaltige Verhältnisse. Was nun die erstere Reihe meiner Beobachtungen anbetrifft, so umfaßt dieselbe 812 Tripperfälle, von denen 91 durch Nebenhodenentzündungen kompliziert

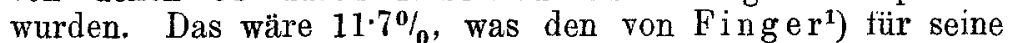
Poliklinik gefundenen $12.5 \%$ and ebenso den $12 \%$ von $\mathrm{Le}$ Clerc Daudry ${ }^{2}$ genau entsprechen würde. Andere Autoren haben freilich teils niedrigere, teils höhere Zahlen erhalten, so z. B. Gebert ${ }^{3}$ für die Blaschkosche Poliklinik 7\%, B e r g $\left.{ }^{4}\right) \quad 7 \cdot 5 \%$ und W a g a p w ${ }^{5}$ ) $8.4 \%$. Die beiden letzteren in der Privatpraxis. Höhere Prozentzahlen als Finger, Daudry und ich führen beispielsweise Pezzoli und Porges ${ }^{6}$ ) an, welche, nach einem im Jahre 1903 erschienenen Bericht über die Tätigkeit des Fingerschen Ambulatoriums, unter 3934 Fällen von unkomplizierter und

1) Finger. Die Blennorrhoe der Sexualorgane. Leipzig und Wien 1901. pag. 293.

$\left.{ }^{2}\right) \mathrm{L}$ e Clerc Daudry. Über Epididymitis gonorrh. Journal méd. de Bruxelles 1901. Nr. 32. Ref. München med. W. 1902. pag. 496.

3) Gebert. Therap Mitteilungen. Ther. Monatsh. 1893. pag. 169.

4) B erg, zit. uach Finger l. c.

5) Waga pow. 103 Fälle von Epididymitis ac. Sitzungsber. der Moskauer venerol. dermatol. Ges. 1896. Bd. IV. pag. 2.

6) Pezzoli u. Porges. 12,000 Fälle von Haut- u. Geschlechtskr. Bericht aus Prof. Fingers Ambulatorium Leipzig und Wien 1903. p. 77. 
komplizierter Urethritis 560 Epididymitisfälle hatten, was. $14 \cdot 2 \%$ gleichkommen würde. Noch größer ist das Verhältnis in dem Moskauer Stadtambulatorium ${ }^{1}$ ) für vener. Krankheiten, wo Tichomirow, Pusenkin und Smirnow in den Jahren 1897 bis 1901 inklusive 2336 Tripperfälle mit 406 Nebenhoden- resp. Hodenentzïndungen beobachteten, was $17 \cdot 3 \%$ ausmacht. So viel höher diese Ziffer als die meinige ist, ergänzt sie letztere, weil sie die Verhältnisse unter der ärmsten Bevölkerung Moskaus wiedergiebt. Berücksichtigen wir nun weiter aber auch die Anamnese, so gaben von meinen 812 Tripperkranken, unter denen 363 Personen zum ersten Male einen solchen hatten und 449 bereits mehrfach daran erkrankt waren, 135 Kranke an, daß sie bereits Hodenschwellungen gehabt hätten. Es wird dadurch die von mir beobachtete Zahl von 91 Nebenhodenentzündungen um 135 Fälle erhöht, wodurch die gefundene Verhältniszahl 11.7 auf $27.8 \%$ steigt. So gewaltig hoch diese Ziffer erscheint, entspricht sie doch überraschend genau den Hospitalverhältnissen, denn es fanden in solchen: J. Simonis $\left.\mathrm{s}^{2}\right) 27 \cdot 5 \%$, Roll et t $\left.^{3}\right) 27 \cdot 9 \%$, W e b e $\mathbf{r}^{4}$ ) $28 \cdot 2 \%$, Fing er $)^{5}$ 29.9\% und Baraczynski gar $32 \%$ Epididymitiden. Nur Tarnows $\mathrm{ky}^{6}$ ) und $\mathrm{Jullien}^{7}$ ) geben ganz niedrige Prozentzahlen für die unter ihnen stehenden Hospitäler an, nämlich $12 \cdot 2$ resp. $15 \cdot 2 \%$, also nicht mehr, als andere in der ambulatorischen Praxis sahen. Auffallenderweise stimmt Tarnowskys, für Petersburg gefundene, Angabe genau überein mit der Anzahl der Epididymitisfälle unter den Tripperkranken der Moskauer Stadthospitäler, wie sie im städtischen Bericht für das Jahr $1900^{8}$ ) verzeichnet ist, nämlich 77 Epididymitisfälle unter 657 Trippererkrankungen oder $11.7 \%$. Leider liegt eine derartige Mitteilung für die Moskauer Stadthospitäler nur für dieses eine Jahr vor, indem

1) Berichte üher die Tätigkeit der Moskauer Stadtärzte für die Jahre 1897, 1898, 1899, 1900 u. 1901. Moskau.

2) Zit. nach M. J os e ph. Lehrb. der Hant- u. Geschlechtskr. 1901. Bd. II. pag. 385 .

3) Zit. nach Finger l. c.

4) Weber. Beiträge zur Häufgkeit der Tripperkomplikation beim.

Manne. Inaug.-Dissert. Würzburg 1890.

5) Fing er 1. c.

$\left.{ }^{8}\right)$ Ref. nach Dermatol. Zeitsch. 1903. pag. 306.

7) Fing er 1. c.

8) Bericht über die Anstalten der allg. Fürsorge, welche sich unter Aufsicht der Moskauer Stadtverwaltung befinden, für das Jahr 1900. Moskau 1901. 
bei den späteren Berichten die gonorrhoischen Epididymitiden nicht mehr getrennt von den Tripperfällen angegeben werden. Eine Erklärung können diese auBerordentlich niedrigen Verhältniszahlen nur dadurch finden, daß in Petersburg, Moskau und auch Paris die Tripperkranken der ärmeren Klassen nicht nur ausschließlich bei eingetretenen Komplikationen das Krankenhaus aufsuchen müssen.

Zum Vergleich und andererseits zu noch vielseitigerer Beleuchtung der uns interessierenden Frage zog ich, wie schon gesagt, auch alle meine sonstigen Patienten in den Kreis der Betrachtung. Es erwies sich, daß unter 957 Kranken mit verschiedenen Haut- und Geschlechtsleiden, außer Tripper, im Alter von über 19 Jabren, 493 einen solchen gehabt zu haben verneinten und 464 einen solchen bejahten. Von letzteren wiederum erklärten auf meine Frage, nach dem Vorkommen einer schmerzhaften, mit Hitze verlaufenen Hodenschwellung, 152 eine solche gehabt zu haben. Das macht 32.7\% Epididymitiden. Obgleich diese Ziffer alle bisher angegebenen übertrifft, findet sie doch ein Analogon in den $32 \%$ Nebenhodenentzündungen bei Gonorrhoe, welche, wie erwähnt, B ar ac z y n s k i im Rudolfspital zu Wien beobachtet hat. Wenn meiner letztgenannten Zahl auch nur ein approximativer Wert beizulegen ist, wirft sie doch ein ernstes Licht auf die Bedeutung des Trippers in sozialer Hinsicht, indem $48 \%$ meiner Patienten einen Tripper gehabt zu haben zugaben, von denen $1 / 3$ durch eingetretene Hodenentzündungen dauernd geschädigt worden waren. Beiläufig sei bemerkt, daß - nach einer jüngst erschienenen Statistik von $\mathrm{E} \mathbf{r ~ b}^{1}$ ) - unter 600 männlichen Patieuten seiner Klientel, über 25 Jahren, $50 \%$ einen Tripper gehabt hatten. In Japan scheinen die Verhältnisse noch schlimmer zu sein, indem nach einer Statistik ron $0 \mathrm{ka} \mathrm{mu} \mathrm{ra}{ }^{2}$ ) $60-70 \%$ der erwachsenen Männer an Blennorhoe leiden.

Nach dieser Abschweitung kehre ich wieder zur Betrachtung der persönlich von mir beobachteten 91 Fälle von Nebenhodenentzündung zuräck. Hinsichtlich ihrer Lokalisation gilt, daß es sich 49 mal um den linken, 32 mal um den rechten und 10 mal um beide Nebenhoden handelte. Ahnlich war das Verhältnis bei den 135 Tripperkranken, welche, ihren Worten gemäß, bereits Hodenschwellungen gehabt hatten, indem dieselbe 55 mal auf der linken, 45 mal auf der rechten, 29 mal auf beiden und $6 \mathrm{mal}$ unbekannt auf welcher Seite gewesen sein sollte. Es überwiegt somit, nach meinen Er-

I) Er b. Syphilis u. Tabes. Berl, klin. W. 1904. pag. 41.

2) Okamura. Die Verbreitung der vener. Kr. Ref. Monatsh. f. prakt. Dermatol. 1904. Bd. XXXVIII. pag. 346. 
fahrungen, die linksseitige Nebenhodenentzündung ein wenig über die rechtsseitige, wenn auch die Unterschiede nur geringe sind. Das widerspricht den Angaben einzelner Autoren, wie Finger, Max Joseph, Gebert etc., welche ein geringes Überwiegen der rechten Seite fanden, im übrigen sich aber dahin aussprechen, daß beide Seiten fast gleich häufig erkranken. Völlig einverstanden erkläre ich mich mit der allgemeinen Ansicht, daß ein gleichzeitiges Befallenwerden beider Nebenhoden zu den größten Ausnahmen gehört. In allen meinen derartigen Fällen ließ sich stets ein Nacheinander der Erkrankung beider Nebenhoden sicher feststellen. Gewöhnlich erkrankt der zweite Nebenhoden einige Tage bis Wochen später. In einigen Fällen beobachtet man, da $\beta$ die Patienten bei neuen Trippererkrankungen immer wieder auf ein und derselben Seite die Nebenhodenentzündung bekommen, in anderen dagegen, daß3 bald der eine, bald der andere Nebenhoden erkrankt. Dieses alles spricht entschieden dafür, daß beide Nebenhoden gleicherweise zur Erkrankung neigen. Unter den äußeren Ursachen, welche eine Erkrankung begünstigen, denkt man unwillkürlich vor allem an den Beruf. Meine Erfahrung aber lehrt, daß die Nebenhodenentzündung so ziemlich bei jeder Berufsart vorkommen kann, ja daß sie häufig dort vermißt wird, wo sie dem Beruf nach zu erwarten wäre. Es kommt nicht so sehr auf die Beschäftigung an, als auf die Möglichkeit, sich während des Trippers vor forzierten $\mathrm{Be}-$ wegungen, wie Sturz, Sprung, Stoß etc. schützen zu können. Weiter spielen der Coitus und vor allem instrumentelle Eingriffe nicht nur im akuten, sondern auch im chronischen Stadium eine maßgebende Rolle. Unter den instrumentellen Eingriffen sind vor allem Bougierungen zu nennen, aber auch Tietspritzungen und Jan etsche Waschungen können dieselbe unangenehme Folge zeitigen. Ist es aber einmal zu einer Epididymitis gekommen, dann genügt eine unvorsichtige Bewegung im Bett oder die stärkere Anwendung der Bauchpresse, um eine Erkrankung auch des anderen Nebenhodens herbeizuführen.

Über das A lte $r$ meiner Tripperkranken und derjenigen von ihnen, welche Nebenhodenentzündungen hatten, geben folgende Tabellen Aufschluß: 
Alter der Tripperkranken:

\begin{tabular}{|c|c|c|c|c|c|c|c|c|c|c|c|}
\hline $\begin{array}{c}\text { Unter } \\
16 \text { Jahren }\end{array}$ & 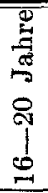 & 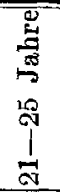 & 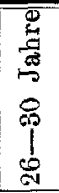 & 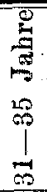 & 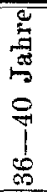 & 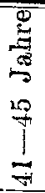 & 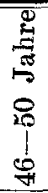 & 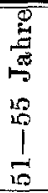 & $\begin{array}{l}0 \\
0 \\
0 \\
0 \\
0 \\
0 \\
0 \\
0 \\
0\end{array}$ & 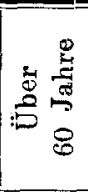 & 葛 \\
\hline 2 & 96 & 268 & 285 & 99 & 47 & 34 & 17 & 7 & 5 & 2 & 812 \\
\hline
\end{tabular}

Alter der Tripperkranken mit Epididymitis :

\begin{tabular}{|c|c|c|c|c|c|c|c|c|c|c|c|}
\hline $\begin{array}{c}\text { Unter } \\
16 \text { Jahren }\end{array}$ & 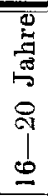 & 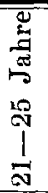 & 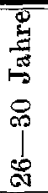 & 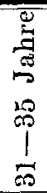 & 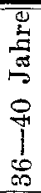 & 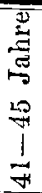 & $\begin{array}{l}0 \\
0 \\
0 \\
0 \\
0\end{array}$ & 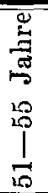 & 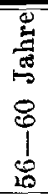 & م. & 荧 \\
\hline 1 & 12 & 31 & 20 & 11 & 5 & 4 & 4 & 2 & 1 & - & 91 \\
\hline
\end{tabular}

Nach diesen Tabellen ist das Alter von keinem nennenswerten Einfluß auf die Nebenhodenentzündung, es sei denn vielleicht, daß einerseits sehr jugendliche Patienten, welche weniger als 16 Jahre zählen und andererseits die über 45 jährigen Tripperkranken verhältnismäßig öfter von dieser Komplikation befallen werden. Die Zahlen sind aber so klein, daß sie sich nicht zu irgendwelchen Schlüssen eignen. Es ist früher einmal von Fing er, ${ }^{\mathbf{1}}$ ) auf Grund von Beobachtungen an der Zeisslschen Klinik, die Vermutung ausgesprochen worden, daß die Jahreszeiten für die Entstehung der Nebenhodenentzïndung Bedeutung haben, insofern als das Maximum derselben, in die Zeit der anhaltenden Trockenheit und bedeutendsten Hitze* fallen soll. Eine Bestätigung dieser

1) S chmidts Jahrbächer 1882. Bd. CXCIII. pag. 202. 
Vermutung haben meine Fälle nicht gebracht und auch sonst scheint dieselbe, soweit ich die Literatur verfolgen konnte, keinen Anklang gefunden zu haben.

Dagegen ergaben meine Beobachtungen einen entschiedenen Einfluß einer bestimmten Zeitperiode des Trippers auf das Zustandekommen der Nebenhodenentzündung, wie folgende Tabelle beweist:

Zeit des Auftretens der Epididymitis nach der Dauer des Trippers :

In der :

In dem :

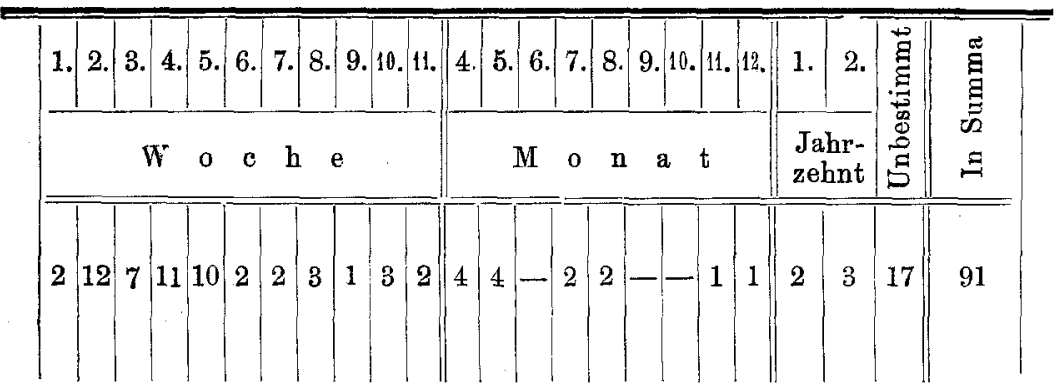

Das Maximum der Nebenhodenentzündungen fällt demnach auf die 2.-5. Woche des akuten, resp. subakuten Trippers oder in die Zeit, wo der Tripper auf die p. posterior überzugehen pflegt. Seltener kommt die Epididymitis im chr. Stadium vor und nur ausnahmsweise nach dem ersten Jahr. Sehr merkwürdig ist das Auftreten von Epididymitis Jahrzehnte nach dem Tripper bei bestehenden Resten desselben. Ob nicht in diesen Fällen die beginnende Prostatahypertrophie verhängnisvoll wird, indem durch das stärkere Drängen und Pressen beim Urinieren der Blutzufluß zu den Geschlechtsorganen gesteigert wird und eventuell eingekapselte Gonokokken zum Aufleben kommen. Lucas, ${ }^{1}$ ) $\mathrm{Colombin} \mathrm{i}^{2}$ ) und andere haben gefunden, daß die gonorrhoische Epididymitis überhaupt

1) G. Lu cas. Résultats de toucher rectal dans 285 cas d'épididymites blennorrhagiques. Annal. de dermatol. et de syphiligr. 1894. p. 1157 .

2) Colombini. Über die Häufigkeit der Prostatitis, Vesiculitis etc. Ref. Monatsh. f. prakt. Dermatol. 1896. Bd. XXIII. pag. 49. 
oft mit Prostatitis verbunden ist, nämlich in 64 resp. $62.5 \%$. Lu cas gibt außerdem an, daß die Veränderungen der Prostata fast immer auf derjenigen Hälfte der Vorsteherdrüse zu konstatieren sind, welche der Nebenhodenentzündung entspricht. Leider habe ich versäumt, alle meine Epididymitisfälle auf diesen Punkt hin zu untersuchen, kann daher nur im allgemeinen bestätigen, daß Veränderungen der Prostata die Epididymitis begleiten. Dieselben waren aber, nach meinen Erfahrungen, stets nur geringfügiger Natur. Keinmal habe ich eine akute parenchymatöse Prostatitis bei Epididymitis gesehen. Veränderungen der Prostata bei Epididymitis sind mehr als natürlich, wo das Zustandekommen einer Nebenhodenentzïndung beim Tripper doch gewiß durch ein Weitergehen des Prozesses von der Urethra zu den höher gelegenen Organen zu erklären ist. Mitbeteiligungen des Samenstranges in bald stärkerem, bald schwächerem Grade gehören daher zur Regel. Baermanns $\mathbf{s}^{\mathbf{1}}$ ) Behauptung, daß jede Epididymitis, die im Verlauf einer gonorrhoischen Urethritis auftritt, auf eine Gonokokkeninvasion zurückzuführen ist, hat viel Wahrscheinlichkeit für sich.

Überblicken wir kurz das Gesagte:

1. Epididymitis gonorrhoica beobachtete ich in $11.7 \%$ aller ambulatorisch behandelten Tripperfälle.

2. Bei Berücksichtigung der Anamnese ergab sich aber, daß bei denselben Kranken die Verhältniszahl der Epididymitis zum Tripper $27 \cdot 8 \%$ beträgt.

3. Die von verschiedenen Autoren für die Spitalpraxis gefundene Ziffer von ca. $30 \%$ gonorrhoischer Epididymitis scheint daher der Gesamtrerhältniszahl der Epididymitis gonorrhoica näher zu kommen als diejenige der ambulanten Praxis.

4. Beide Nebenhoden erkranken gleich häufig, jedoch selten gleichzeitig und dann stets nacheinander und nicht auf einmal.

1) Baermann. Über die Pathogenese der gonorrhoischen Epididymitis etc. Deutsche med. W. 1903. pag. 720 . 
5. Der Beruf, das Alter der Patienten und die Jahreszeiten sind von keinem nennenswerten Einfluß für das Entstehen der Epididymitis.

6. Forzierte Bewegung, Coitus, instrumentelle Eingriffe sind die häufigsten Ursachen.

7. Die meisten Erkrankungen an Epididymitis gonorrhoica fallen in die 2. -5 . Woche des akuten, resp. subakaten Trippers. 